\title{
A Family of Global Attractors for the Higher-order Kirchhoff-type Equations and Its Dimension Estimation
}

\author{
Guoguang Lin *, Yuhang Chen \\ School of Mathematics and Statistics, Yunnan University, Kunming, China \\ Email address: \\ gglin@ynu.edu.cn (Guoguang Lin), 732517411@qq.com (Yuhang Chen) \\ ${ }^{*}$ Corresponding author
}

\section{To cite this article:}

Guoguang Lin, Yuhang Chen. A Family of Global Attractors for the Higher-order Kirchhoff-type Equations and Its Dimension Estimation. American Journal of Applied Mathematics. Vol. 8, No. 6, 2020, pp. 300-310. doi: 10.11648/j.ajam.20200806.12

Received: November 4, 2020; Accepted: November 16, 2020; Published: November 24, 2020

\begin{abstract}
In this paper, we study the long-time behavior of solutions for a class of initial boundary value problems of higher order Kirchhoff -type equations, and make appropriate assumptions about the Kirchhoff stress term. We use the uniform prior estimation and Galerkin method to prove the existence and uniqueness of the solution of the equation, when the order $m$ and the order q meet certain conditions. Then, we use the prior estimation to get the bounded absorption set, it is further proved that using the Rellich-Kondrachov compact embedding theorem, the solution semigroup generated by the equation has a family of global attractor. Then the equation is linearized and rewritten into a first-order variational equation, and it is proved that the solution semigroup is Frechet differentiable. Finally, it proves that the Hausdorff dimension and Fractal dimension of a family of global attractors are finite.
\end{abstract}

Keywords: Kirchhoff-Type Equation, Prior Estimation, Galerkin Method, A family of Global Attractors, Hausdorff Dimension, Fractal Dimension

\section{Introduction}

In this paper, we considers the initial-boundary value problem of the following higher-order nonlinear Kirchhoff-type equations

$$
\begin{gathered}
u_{t t}+a(t)(-\Delta)^{m} u_{t}+\Delta^{2 m} u+N\left(\left\|D^{m} u(t)\right\|_{q}^{q}\right)(-\Delta)^{m} u=I(x), \\
u(x, t)=0, \frac{\partial^{i} u}{\partial v^{i}}=0, i=1,2, \cdots, 2 m-1, x \in \partial \Omega, t>0, \\
u(x, 0)=u_{0}(x), u_{t}(x, 0)=u_{1}(x), x \in \Omega \subset R^{n} .
\end{gathered}
$$

Where $\Omega$ is a bounded domain in $R^{n}(n \geq 1)$ with smooth boundary $\partial \Omega, m \in N^{+}, \partial \Omega$ is the homogeneous Dirichlet boundary of $\Omega, v$ is an outer normal vector of $\partial \Omega, a(t)$ is a general function of $\mathrm{t}$, and $I(x)$ is an external force term. The assumptions about $a(t)$ and $N\left(\left\|D^{m} u(t)\right\|_{q}^{q}\right)$ will be given later.
In 1883, Gustav Robert Kirchhoff [1], a German physicist, first introduced the following equation when he studied the transverse vibration of stretched strings

$$
\rho h \frac{\partial^{2} u}{\partial t^{2}}-\left\{P_{0}+\frac{E h}{2 L} \int_{0}^{L}\left(\frac{\partial u}{\partial t}\right)^{2} d x\right\} \frac{\partial^{2} u}{\partial x^{2}}=0,0<x<L, t \geq 0,
$$

Where $\mathrm{h}$ is the cross-sectional area of the string, $\mathrm{E}$ is young coefficient, $P_{0}$ is the initial axial tension, $\mathrm{L}$ is the length of the string, $\rho$ is the mass density of the string, and $u=u(x, t)$ is the lateral displacement in the spatial axial coordinate. Over the past a hundred years, with the development of science and technology, Kirchhoff equation has been paid more and more attention by scholars. The application field of Kirchhoff equation is also expanding, and its expression is constantly extended. A series of mathematical theories and research results have been obtained, such as the existence and uniqueness of global solution, the decay of solution, the existence of random attractor, and the index Numerical attractor, global attractor and its dimension estimation, inertial manifold, etc [2-6]. 
When $\mathrm{m}=1, \mathrm{q}=2$, the stress term is $\left(1+\|\nabla u(t)\|_{2}^{2}\right)$, and a nonlinear nonlocal source term is added to equation (1), Mitsuhiro Nakao [7] studied the existence of attractors and some absorption properties in the local sense for this class of Kirchhoff type quasilinear wave equation with standard dissipation term $u_{t}$. Then, Zaiyun Zhang [8] and others studied the initial boundary value problem of nonlinear dissipative Kirchhoff equation when $\mathrm{m}=0, \mathrm{q}=2, a(t)=\beta$, $N(s)=M\left(\|u(t)\|^{2}\right)$, the initial boundary value problem of nonlinear dissipative Kirchhoff equation is as follows

$$
u_{t t}+M\left(\|u\|^{2}\right) \Delta u+\beta u_{t}+g(u)=f(x),
$$

In Hilbert spaces $H_{0}^{1}(\Omega) \times L^{2}(\Omega)$ and $D(A) \times H_{0}^{1}(\Omega)$, they estimate the dimensions of global attractors by using rescaling technique and linearized variational method. The upper bounds of Hausdorff dimension and Fractal dimension are obtained.

Furthermore, when $\mathrm{m}=1, \mathrm{q}=2$, stress term $N(s)=\varphi\left(\|D u\|^{2}\right)$ and strong dissipation term $a(t)=\sigma\left(\|D u\|^{2}\right)$, chueshov Igor [9] studied the well posedness and long-time behavior of the solution of the initial boundary value problem of the equation

$$
u_{t t}-\sigma\left(\|D u\|^{2}\right) \Delta u_{t}-\varphi\left(\|D u\|^{2}\right) \Delta u+g(u)=h(x),
$$

By assuming the Kirchhoff term, he proved the existence and uniqueness of weak solution and the existence of a finite dimensional global attractor in the natural energy space with partial strong topology, and further proved that the attractor is strong under non supercritical conditions.

Recently, on the basis of chueshov Igor [9], Guoguang Lin [10] and others studied the long-term behavior of the initial boundary value problem for a class of nonlinear strongly damped higher order Kirchhoff type equation with $m=1, q=2, a(t)=1$ and $N\left(\left\|D^{m} u(t)\right\|_{2}^{2}\right)=\left(\left\|D^{m} u(t)\right\|_{2}^{2}\right)^{q}$

$$
u_{t t}+(-\Delta)^{m} u_{t}+\left\|\nabla^{m} u\right\|^{2 q}(-\Delta)^{m} u+g(u)=f(x) \text {, }
$$

They obtain the existence and uniqueness of the solution and the global attractor, and consider the dimension of the global attractor and the upper bound estimation of the dimension. For more related research results on the Kirchhoff equation, please refer to [11-15].

In this paper, based on the long-time behavior of solutions of some nonlinear Kirchhoff type equations with initial boundary value problems, a class of higher order Kirchhoff type equations with the highest order term $\Delta^{2 m} u\left(m \in N^{+}\right)$is studied. Because of the uncertainty of $\mathrm{m}$ in Kirchhoff stress term $N\left(\left\|D^{m} u(t)\right\|_{q}^{q}\right)$ and $q$ in Banach space $L^{q}(\Omega)$, there will be a bottleneck when using uniform prior estimation and Galerkin finite element method to prove the existence and uniqueness of the global solution of the equation, so it is impossible to continue the follow-up work. We get a relation between $\mathrm{m}$ and $\mathrm{q}$ in Banach space $L^{q}(\Omega)$ by the theory of Sobolev space. Therefore, we overcome this problem successfully and get more extensive research methods and theoretical results.

\section{Preliminaries}

For brevity, we used the follow abbreviation:

$$
H=L^{2}(\Omega), D=\nabla,\|\cdot\|_{L^{2}}=\|\cdot\|, H_{0}^{m}(\Omega)=H^{m}(\Omega) \cap H_{0}^{1}(\Omega),
$$
$H_{0}^{2 m+k}(\Omega)=H^{2 m+k}(\Omega) \cap H_{0}^{1}(\Omega), E_{k}=H_{0}^{2 m+k}(\Omega) \times H_{0}^{k}(\Omega)$, Let $A_{k}$ be a family of global attractors from $E_{0}$ to $E_{k}$, and $B_{0 k}$ be a bounded absorbing set in $E_{k}$, with $k=0,1,2,3, \cdots, 2 m$ and $C_{i}(i=1,2,3, \cdots)$ denotes positive constant. The notation $(\cdot, \cdot),\|\cdot\|$ for the $H$ inner product and norm, that is $(u, v)=\int_{\Omega} u(x) v(x) d x,(u, u)=\|u\|^{2}$.

Now we state some assumptions and result. Suppose the functions $a(t)$ and $N\left(\left\|D^{m} u(t)\right\|_{q}^{q}\right)$ in the equation satisfy the following conditions.

(A1) $a(t) \in C^{2}[0, \infty)$ and $\forall t \geq 0$, such that

$$
2+\varepsilon \leq \mu_{0} \leq a(t) \leq \mu_{1}, \mu= \begin{cases}\mu_{0} & \frac{d}{d t}\left\|D^{m+k} u\right\|^{2} \geq 0 \\ \mu_{1} & \frac{d}{d t}\left\|D^{m+k} u\right\|^{2}<0\end{cases}
$$

(A2) $N(s) \in C^{2}[0, \infty)$ and $\forall s \geq 0$, such that

$$
1+\varepsilon \leq \delta_{0} \leq N(s) \leq \delta_{1}, \delta= \begin{cases}\delta_{0} & \frac{d}{d t}\left\|D^{m+k} u\right\|^{2} \geq 0 \\ \delta_{1} & \frac{d}{d t}\left\|D^{m+k} u\right\|^{2}<0\end{cases}
$$

Where

$$
\begin{gathered}
0<\varepsilon \leq \min \left\{\frac{\sqrt{1+2 \mu_{1} \lambda_{1}^{m}}-1}{2}, \frac{\delta}{\mu}, \frac{2 \delta_{0}}{\lambda_{1}^{-m}+2 \mu_{0}}, \frac{\delta_{1}}{\mu_{0}}, \frac{\sqrt{1+\mu_{0} \lambda_{1}{ }^{m}}-1}{2}, \frac{2 \delta_{0}}{\mu_{1}+\lambda_{1}^{-m}}\right\} \\
\text { (A3) }\left\{\begin{array}{l}
\frac{2 n}{n+2 m} \leq q \leq \frac{2 n}{n-2 m}, \quad n>2 m ; \\
\frac{2 n}{n+2 m} \leq q<\infty, \quad n \leq 2 m .
\end{array}\right.
\end{gathered}
$$

\section{The Existence of a Family of Global Attractors}

Lemma 1 Suppose that the $(A 1)-(A 2)$ are satisfied, and $I(x) \in H, \quad\left(u_{0}, u_{1}\right) \in E_{k}$. Then the initial boundary value problem (1) - (3) has a global solution $(u, v)$ satisfies 


$$
\begin{array}{cc}
u \in L^{\infty}\left(0,+\infty ; H_{0}^{2 m+k}(\Omega)\right), & y_{1}(0)=\left\|D^{k} v_{0}\right\|^{2}+\left\|D^{2 m+k} u_{0}\right\|^{2}+\delta\left\|D^{m+k} u_{0}\right\|^{2} . \\
v \in L^{\infty}\left(0,+\infty ; H_{0}^{k}(\Omega)\right) \cap L^{2}\left(0, T ; H_{0}^{m+k}(\Omega)\right), \text { and } & \text { Thus, there exists a non-negative constant } R_{k} \text { and } \\
\|(u, v)\|_{E_{k}}^{2}=\left\|D^{2 m+k} u\right\|^{2}+\left\|D^{k} v\right\|^{2} \leq y_{1}(0) e^{-\gamma_{1} t}+\frac{C_{1}}{\gamma_{1}}\left(1-e^{-\gamma_{1} t}\right), & t_{k}=t_{k}(\Omega)>0, \text { such that } \\
\mu_{0} \int_{0}^{T}\left\|D^{m+k} v\right\|^{2} d t \leq y_{1}(0)+\int_{0}^{T} C_{1} d t, & \|(u, v)\|_{E_{k}}^{2}=\left\|D^{2 m+k} u\right\|^{2}+\left\|D^{k} v\right\|^{2} \leq R_{k}^{2} \quad\left(t>t_{k}\right) . \\
\text { where } v=u_{t}+\varepsilon u, \quad \gamma_{1}=\min \left\{k_{1}, \frac{k_{2}}{\delta}, 2 \varepsilon\right\}, & \text { Proof. Let }(-\Delta)^{k} v=(-\Delta)^{k} u_{t}+\varepsilon(-\Delta)^{k} u \text {, by taking the inner } \\
\left(u_{t t}+a(t)(-\Delta)^{m} u_{t}+\Delta^{2 m} u+N\left(\left\|D^{m} u(t)\right\|_{q}^{q}\right)(-\Delta)^{m} u,(-\Delta)^{k} v\right)=\left(I(x),(-\Delta)^{k} v\right) .
\end{array}
$$

By using Hölder Inequality, Young inequality and Poincaré inequality, the following are obtained by dealing with the following items in formula (4)

$$
\left(u_{t t},(-\Delta)^{k} v\right) \geq \frac{1}{2} \frac{d}{d t}\left\|D^{k} v\right\|^{2}-\frac{2 \varepsilon+\varepsilon^{2}}{2}\left\|D^{k} v\right\|^{2}-\frac{\varepsilon^{2} \lambda_{1}^{-m}}{2}\left\|D^{m+k} u\right\|^{2}
$$

By assuming (A1) and using Young inequality and Poincaré inequality to deal with the strong damping term, we can obtain

$$
\begin{gathered}
\left(a(t)(-\Delta)^{m} u_{t},(-\Delta)^{k} v\right) \geq \frac{\mu_{0}}{2}\left\|D^{m+k} v\right\|^{2}-\frac{\varepsilon^{2} \mu_{1}}{2}\left\|D^{m+k} u\right\|^{2}, \\
\left(\Delta^{2 m} u,(-\Delta)^{k} v\right)=\left(\Delta^{2 m} u,(-\Delta)^{k}\left(u_{t}+\varepsilon u\right)\right)=\frac{1}{2} \frac{d}{d t}\left\|D^{2 m+k} u\right\|^{2}+\varepsilon\left\|D^{2 m+k} u\right\|^{2}
\end{gathered}
$$

It can be obtained from hypothesis (A2)

$$
\left(N\left(\left\|D^{m} u(t)\right\|_{q}^{q}\right)(-\Delta)^{m} u,(-\Delta)^{k} v\right) \geq \frac{\delta}{2} \frac{d}{d t}\left\|D^{m+k} u\right\|^{2}+\varepsilon \delta_{0}\left\|D^{m+k} u\right\|^{2} .
$$

Using Schwarz inequality and Young inequality to deal with the external force term, we obtain

$$
\left(I(x),(-\Delta)^{k} v\right)=\left(D^{k} I(x), D^{k} v\right) \leq \frac{1}{2 \varepsilon^{2}}\left\|D^{k} I(x)\right\|^{2}+\frac{\varepsilon^{2}}{2}\left\|D^{k} v\right\|^{2} \leq C_{1}+\frac{\varepsilon^{2}}{2}\left\|D^{k} v\right\|^{2} .
$$

Substituting (5) - (9) into (4), we receive

$$
\begin{gathered}
\frac{d}{d t}\left(\left\|D^{k} v\right\|^{2}+\left\|D^{2 m+k} u\right\|^{2}+\delta\left\|D^{m+k} u\right\|^{2}\right)+\left(\frac{\mu_{0} \lambda_{1}^{m}}{2}-2 \varepsilon-2 \varepsilon^{2}\right)\left\|D^{k} v\right\|^{2}+2 \varepsilon\left\|D^{2 m+k} u\right\|^{2} \\
+\delta\left(\frac{2 \varepsilon \delta_{0}-\varepsilon^{2} \lambda_{1}^{-m}-\varepsilon^{2} \mu_{1}}{\delta}\right)\left\|D^{m+k} u\right\|^{2}+\mu_{0}\left\|D^{m+k} v\right\|^{2} \leq C_{1} .
\end{gathered}
$$

According to hypothesis (A1), we have

$$
\begin{gathered}
k_{1}=\frac{\mu_{0} \lambda_{1}^{m}}{2}-2 \varepsilon-2 \varepsilon^{2} \geq 0, k_{2}=2 \varepsilon \delta_{0}-\varepsilon^{2} \lambda_{1}^{-m}-\varepsilon^{2} \mu_{1} \geq 0 . \\
\text { Let } \gamma_{1}=\min \left\{k_{1}, \frac{k_{2}}{\delta}, 2 \varepsilon\right\}, \text { then we get } \\
\frac{d}{d t} y_{1}(t)+\gamma_{1} y_{1}(t)+\mu_{0}\left\|D^{m+k} v\right\|^{2} \leq C_{1},
\end{gathered}
$$

where

$$
y_{1}(t)=\left\|D^{k} v\right\|^{2}+\left\|D^{2 m+k} u\right\|^{2}+\delta\left\|D^{m+k} u\right\|^{2}
$$

By using Gronwall inequality, we obtain

$$
y_{1}(t) \leq y_{1}(0) e^{-\gamma_{1} t}+\frac{C_{1}}{\gamma_{1}}\left(1-e^{-\gamma_{1} t}\right),
$$




$$
\mu_{0} \int_{0}^{T}\left\|D^{m+k} v\right\|^{2} d t \leq y_{1}(0)+\int_{0}^{T} C_{1} d t
$$

So, we have

$$
\|(u, v)\|_{E_{k}}^{2}=\left\|D^{2 m+k} u\right\|^{2}+\left\|D^{k} v\right\|^{2} \leq y_{1}(0) e^{-\gamma_{1} t}+\frac{C_{1}}{\gamma_{1}}\left(1-e^{-\gamma_{1} t}\right),
$$

and

$$
\varlimsup_{t \rightarrow \infty}\|(u, v)\|_{E_{k}}^{2} \leq \frac{C_{1}}{\gamma_{1}}=R_{k}^{2} \quad\left(t>t_{k}\right) .
$$

Thus, there exist a non-negative constant $R_{k}^{2}$ and $t_{k}=t_{k}(\Omega)>0$, such that

$$
\|(u, v)\|_{E_{k}}^{2}=\left\|D^{2 m+k} u\right\|^{2}+\left\|D^{k} v\right\|^{2} \leq R_{k}^{2} \quad\left(t>t_{k}\right) .
$$

Lemma 1 is proved

Theorem 1 Under the hypotheses of Lemma 1, and $I(x) \in H, \quad\left(u_{0}, u_{1}\right) \in E_{k}$. So the initial boundary value problem (1) - (3) has a unique global solution $(u, v) \in L^{\infty}\left([0,+\infty) ; E_{k}\right)$.

Proof. Existence: the existence of global solution is proved by Galerkin method

Step 1: construct approximate solution

Let $(-\Delta)^{m+z} w_{j}=\lambda_{j}^{m+z} w_{j}, z=0,1,2,3, \cdots \cdots, m$, where $\lambda_{j}$ is the eigenvalue of $-\Delta$ with homogeneous Dirichlet boundary on $\Omega, w_{j}$ is the eigenfunction determined by the corresponding eigenvalue $\lambda_{j}$. all the eigenfunctions $\left\{w_{j}\right\}_{j=1}^{\infty}$ constitute the standard orthogonal basis of $H_{0}^{2 m}(\Omega) \cap H(\Omega)$.

Set $u_{s}=u_{s}(t)=\sum_{j=1}^{s} g_{j s}(t) w_{j}$ be the approximate solution of the initial boundary value problem (1) - (3), where $g_{j s}(t)$ is determined by the following system of differential equations

$$
\left(u_{s t t}(t)+a(t)(-\Delta)^{m} u_{s t}(t)+\Delta^{2 m} u_{s}(t)+N\left(\left\|D^{m} u_{s}(t)\right\|_{q}^{q}\right)(-\Delta)^{m} u_{s}(t), w_{j}\right)=\left(I(x), w_{j}\right) \quad(1 \leq j \leq s)
$$

Where, (18) meet the initial conditions $u_{s}(0)=u_{0 s}$, $u_{s t}(0)=u_{1 s}$.

When $s \rightarrow+\infty$, we can obtain $\left(u_{0 s}, u_{1 s}\right) \rightarrow\left(u_{0}, u_{1}\right)$ in $E_{k}$ According to the basic theory of solutions of ordinary differential equations, we know that the approximate solution $u_{s}(t)$ exists on $\left(0, t_{s}\right)$. Step 2: prior estimation

In order to prove the existence of weak solution in space $E_{k}(k=0,1,2,3, \cdots, 2 m)$, we multiply $g_{j s}^{\prime}(t)+\varepsilon g_{j s}(t)$ at bothsides of equation (18) and sum of $j$, Set $v_{s}(t)=u_{s t}(t)+\varepsilon u_{s}(t)$.

When $k=0,1,2,3, \cdots, 2 m$, we get a priori estimate of solution in space $E_{k}$

$$
\begin{gathered}
\left\|\left(u_{s}, v_{s}\right)\right\|_{E_{k}}^{2}=\left\|D^{2 m+k} u_{s}\right\|^{2}+\left\|D^{k} v_{s}\right\|^{2} \leq R_{k}^{2}, \\
\mu_{0} \int_{0}^{T}\left\|D^{m+k} v\right\|^{2} d t \leq y_{1}(0)+\int_{0}^{T} C_{1} d t .
\end{gathered}
$$

It can be seen that the priori estimates of the solution of lemma 1 in formula (19) and (20) hold respectively. It is known that $\left(u_{s}, v_{s}\right)$ is bounded in $L^{\infty}\left([0,+\infty) ; E_{k}\right)$ by formula (19) and can be obtained $v_{s} \in L^{2}\left(0, T ; H_{0}^{m+k}(\Omega)\right)$ by formula (20).

Step 3: limit process

In space $E_{k}(k=0,1,2,3, \cdots, 2 m)$, the subsequence $\left\{u_{l}\right\}$ is selected from sequence $\left\{u_{s}\right\}$, so that $\left(u_{l}, v_{l}\right) \rightarrow(u, v)$ converges weakly $*$ in $L^{\infty}\left([0,+\infty) ; E_{k}\right)$. In addition, from formula (20), we can get that $u_{l}$ is bounded in $L^{2}\left(0, T ; H_{0}^{m+k}(\Omega)\right)$.

If we know that $E_{k}$ is closely embedded in $E_{0}$ by rellich-kondrachov compact embedding theorem, then $\left(u_{l}, v_{l}\right) \rightarrow(u, v)$ converges strongly almost everywhere in $E_{0}$. Thus, we can make $s=l$ and take the limit in equation (18). For fixed $j$, and $l \geq j$, we can get from formula (18).

$$
\left(u_{l t t}(t)+a(t)(-\Delta)^{m} u_{l t}(t)+\Delta^{2 m} u_{l}(t)+N\left(\left\|D^{m} u_{l}(t)\right\|_{q}^{q}\right)(-\Delta)^{m} u_{l}(t), w_{j}\right)=\left(I(x), w_{j}\right) .
$$

Because $u_{s} \rightarrow u$ weakly * converges in $L^{\infty}\left([0,+\infty) ; H^{2 m+k}(\Omega) \cap H_{0}^{1}(\Omega)\right)$, then

B weakly* converges in $\mathrm{C}$

$$
\begin{gathered}
\left(u_{l}(t),(-\Delta)^{k} w_{j}\right) \rightarrow\left(u(t), \lambda_{j}{ }^{k} w_{j}\right) \text { weakly* converges in } L^{\infty}[0,+\infty), \\
\left(u_{l t}(t),(-\Delta)^{k} w_{j}\right) \rightarrow\left(u_{t}(t), \lambda_{j}{ }^{k} w_{j}\right) \text { weakly* converges in } L^{\infty}[0,+\infty) .
\end{gathered}
$$


Therefore, $\left(u_{l t t}(t),(-\Delta)^{k} w_{j}\right)=\frac{d}{d t}\left(u_{l t}(t),(-\Delta)^{k} w_{j}\right) \rightarrow\left(u_{t t}(t), \lambda_{j}^{k} w_{j}\right)$ converges in $D^{\prime}[0,+\infty), D^{\prime}[0,+\infty)$ is the conjugate space of $D[0,+\infty)$ infinitely differentiable space

$\left(\Delta^{2 m} u_{l}(t),(-\Delta)^{k} w_{j}\right) \rightarrow\left(\Delta^{2 m} u(t), \lambda_{j}^{k} w_{j}\right)$ weakly* converges in $L^{\infty}[0,+\infty)$.

As a result, $\quad\left((-\Delta)^{m} u_{l t}(t),(-\Delta)^{k} w_{j}\right)=\left((-\Delta)^{\frac{k}{2}} v_{l}(t),(-\Delta)^{\frac{2 m+k}{2}} w_{j}\right)-\mathcal{E}\left((-\Delta)^{\frac{m+k}{2}} u_{l}(t),(-\Delta)^{\frac{m+k}{2}} w_{j}\right) \quad, \quad$ then $a(t)\left((-\Delta)^{m} u_{l t}(t),(-\Delta)^{k} w_{j}\right) \rightarrow a(t)\left[\left((-\Delta)^{\frac{k}{2}} v(t), \lambda_{j}{ }^{\frac{2 m+k}{2}} w_{j}\right)-\varepsilon\left((-\Delta)^{\frac{m+k}{2}} u(t), \lambda_{j}^{\frac{m+k}{2}} w_{j}\right)\right]$ weakly* converges in $L^{\infty}[0,+\infty)$.

Futher, $\quad\left(N\left(\left\|D^{m} u_{l}(t)\right\|_{q}^{q}\right)(-\Delta)^{m} u_{l}(t),(-\Delta)^{k} w_{j}\right)=\left(N\left(\left\|D^{m} u_{l}(t)\right\|_{q}^{q}\right)(-\Delta)^{\frac{m+k}{2}} u_{l}(t), \lambda_{j}^{\frac{m+k}{2}} w_{j}\right)$ then $\left(N\left(\left\|D^{m} u_{l}(t)\right\|_{q}^{q}\right)(-\Delta)^{m} u_{l}(t),(-\Delta)^{k} w_{j}\right) \rightarrow\left(N\left(\left\|D^{m} u(t)\right\|_{q}^{q}\right)(-\Delta)^{\frac{m+k}{2}} u(t), \lambda_{j}^{\frac{m+k}{2}} w_{j}\right)$ weakly* converges in $L^{\infty}[0,+\infty)$.

In particular, $u_{0 l} \rightarrow u_{0}$ weakly converges in $E_{k} ; u_{l t} \rightarrow u_{1}$ weakly converges in $E_{k}$.

For any $j$ and $l \rightarrow+\infty$, we can get

$$
\left(u_{t t}(t)+a(t)(-\Delta)^{m} u_{t}(t)+\Delta^{2 m} u(t)+N\left(\left\|D^{m} u(t)\right\|_{q}^{q}\right)(-\Delta)^{m} u(t),(-\Delta)^{k} w_{j}\right)=\left(I(x),(-\Delta)^{k} w_{j}\right) \quad(1 \leq j \leq s) .
$$

Because of the arbitrariness of $w_{j}$, we have for any B $v \in H^{k}(\Omega) \cap H_{0}^{1}(\Omega)$

$$
\left(u_{t t}(t)+a(t)(-\Delta)^{m} u_{t}(t)+\Delta^{2 m} u(t)+N\left(\left\|D^{m} u(t)\right\|_{q}^{q}\right)(-\Delta)^{m} u(t), v\right)=(I(x), v),
$$

Therefore, the existence is proved.

Next, we prove the uniqueness of the solution.

Let $u^{*}, v^{*}$ be two solutions of the system of equations, let $w=u^{*}-v^{*}$, then $w$ satisfies

$$
\begin{gathered}
w_{t t}+a(t)(-\Delta)^{m} w_{t}+\Delta^{2 m} w+N\left(\left\|D^{m} u^{*}(t)\right\|_{q}^{q}\right)(-\Delta)^{m} u^{*}-N\left(\left\|D^{m} v^{*}(t)\right\|_{q}^{q}\right)(-\Delta)^{m} v^{*}=0, \\
w(0)=0, w^{\prime}(0)=0, x \in \Omega \subset R^{n} .
\end{gathered}
$$

By using the inner product in $H$ of equation (1) with $w_{t}+\varepsilon w$, we obtain

$$
\left(w_{t t}+a(t)(-\Delta)^{m} w_{t}+\Delta^{2 m} w+N\left(\left\|D^{m} u^{*}(t)\right\|_{q}^{q}\right)(-\Delta)^{m} u^{*}-N\left(\left\|D^{m} v^{*}(t)\right\|_{q}^{q}\right)(-\Delta)^{m} v^{*}, w_{t}+\varepsilon w\right)=0,
$$

By using Hölder inequality, Young inequality and Poincaré inequality, the following are obtained by dealing with the following items in formula (22)

$$
\begin{gathered}
\left(w_{t t}, w_{t}+\varepsilon w\right)=\frac{1}{2} \frac{d}{d t}\left\|w_{t}\right\|^{2}+\varepsilon \frac{d}{d t}\left(w_{t}, w\right)-\varepsilon\left\|w_{t}\right\|^{2} . \\
\left(\Delta^{2 m} w, w_{t}+\varepsilon w\right)=\frac{1}{2} \frac{d}{d t}\left\|(-\Delta)^{m} w\right\|^{2}+\varepsilon\left\|(-\Delta)^{m} w\right\|^{2} .
\end{gathered}
$$

According to the hypothesis (A1) - (A2), Poincaré inequality and differential mean value theorem, we can obtain

$$
\left(a(t)(-\Delta)^{m} w_{t}, w_{t}+\varepsilon w\right) \geq \frac{\varepsilon \mu}{2} \frac{d}{d t}\left\|D^{m} w\right\|^{2}+\mu_{0}\left\|D^{m} w_{t}\right\|^{2} .
$$

$$
\begin{gathered}
\left(N\left(\left\|D^{m} u^{*}(t)\right\|_{q}^{q}\right)(-\Delta)^{m} u^{*}-N\left(\left\|D^{m} v^{*}(t)\right\|_{q}^{q}\right)(-\Delta)^{m} v^{*}, w_{t}+\varepsilon w\right) \\
=\left(N\left(\left\|D^{m} u^{*}(t)\right\|_{q}^{q}\right)(-\Delta)^{m} u^{*}-N\left(\left\|D^{m} v^{*}(t)\right\|_{q}^{q}\right)(-\Delta)^{m} v^{*}, w_{t}\right) \\
+\left(N\left(\left\|D^{m} u^{*}(t)\right\|_{q}^{q}\right)(-\Delta)^{m} u^{*}-N\left(\left\|D^{m} v^{*}(t)\right\|_{q}^{q}\right)(-\Delta)^{m} v^{*}, \varepsilon w\right) \\
=I_{1}+\varepsilon I_{2} .
\end{gathered}
$$

By using Sobolev embedding theorem, we can obtain that, $H_{0}^{m}(\Omega) \subset L^{q}(\Omega)$, then there is a constant $K>0$, such that

$$
\left\|D^{m} u^{*}(t)\right\|_{L^{q}(\Omega)}^{q} \leq K\left\|(-\Delta)^{m} u^{*}(t)\right\|^{\frac{(2 m+n) q-2 n}{4 m}}\|u\|^{2 m q-n q+2 n} \frac{4 m}{4 m},
$$

where 


$$
\left\{\begin{array}{l}
\frac{2 n}{n+2 m} \leq q \leq \frac{2 n}{n-2 m}, \quad n>2 m ; \\
\frac{2 n}{n+2 m} \leq q<\infty, \quad n \leq 2 m .
\end{array}\right.
$$

Therefore,

$$
\begin{array}{r}
I_{1}=\left(N\left(\left\|D^{m} u^{*}(t)\right\|_{q}^{q}\right)(-\Delta)^{m} u^{*}-N\left(\left\|D^{m} v^{*}(t)\right\|_{q}^{q}\right)(-\Delta)^{m} v^{*}, w_{t}\right) \\
\geq \frac{\delta}{2} \frac{d}{d t}\left\|D^{m} w\right\|^{2}-\left|N^{\prime}\left(\xi_{1}\right)\right|\left(\sum_{i=0}^{q-1}\left\|D^{m} u^{*}(t)\right\|_{q}^{i}\left\|D^{m} v^{*}(t)\right\|_{q}^{q-i-1}\right)\left\|D^{m} w\right\|_{q}\left\|D^{m} v^{*}\right\| D^{m} w_{t} \| \\
\geq \frac{\delta}{2} \frac{d}{d t}\left\|D^{m} w\right\|^{2}-\frac{C_{2}}{4 \mu_{0}}\left\|(-\Delta)^{m} w\right\|^{2}-\mu_{0}\left\|D^{m} w_{t}\right\|^{2} \cdot(30)
\end{array}
$$

Similarly, there are

$$
I_{2} \geq \delta_{1}\left\|D^{m} w\right\|^{2}-\frac{C_{4}}{2}\left\|(-\Delta)^{m} w\right\|^{2}-\frac{C_{3}^{2}}{2}\left\|D^{m} w\right\|^{2}
$$

Combined with formula (24) - (31), it is concluded that

$$
\frac{d}{d t}\left(\left\|w_{t}\right\|^{2}+\left\|(-\Delta)^{m} w\right\|^{2}+(\varepsilon \mu+\delta)\left\|D^{m} w\right\|^{2}+2 \varepsilon\left(w_{t}, w\right)\right) \leq 2 \varepsilon\left\|w_{t}\right\|^{2}
$$

$$
+\left(\frac{C_{2}}{2 \mu_{0}}+\varepsilon C_{4}-2 \varepsilon\right)\left\|(-\Delta)^{m} w\right\|^{2}+\left(\varepsilon C_{3}^{2}-2 \varepsilon \delta_{1}\right)\left\|D^{m} w\right\|^{2} .
$$

Further, due to

$$
\left(w_{t}, w\right) \geq-\frac{1}{2}\left\|w_{t}\right\|^{2}-\frac{\lambda_{1}^{-m}}{2}\left\|D^{m} w\right\|^{2},
$$

so,

$$
\begin{gathered}
\frac{d}{d t}\left(\left\|w_{t}\right\|^{2}+\left\|(-\Delta)^{m} w\right\|^{2}+(\varepsilon \mu+\delta)\left\|D^{m} w\right\|^{2}+2 \varepsilon\left(w_{t}, w\right)\right) \leq 3 \varepsilon\left\|w_{t}\right\|^{2}+\left(\frac{C_{2}}{2 \mu_{0}}+\varepsilon C_{4}-2 \varepsilon\right)\left\|(-\Delta)^{m} w\right\|^{2}+\varepsilon\left(C_{3}^{2}-2 \delta_{1}+\lambda_{1}^{-m}\right)\left\|D^{m} w\right\|^{2}+2 \varepsilon\left(w_{t}, w\right) .(3 \\
\text { Let } \gamma_{2}=\max \left\{3 \varepsilon, \frac{C_{2}}{2 \mu_{0}}+\varepsilon C_{4}-2 \varepsilon, \frac{\varepsilon\left(C_{3}^{2}-2 \delta_{1}+\lambda_{1}^{-m}\right)}{(\varepsilon \mu+\delta)}, 1\right\}, \text { such that } \\
\frac{d}{d t} y_{2}(t) \leq \gamma_{2} y_{2}(t),
\end{gathered}
$$

where

$$
y_{2}(t)=\left\|w_{t}\right\|^{2}+\left\|(-\Delta)^{m} w\right\|^{2}+(\varepsilon \mu+\delta)\left\|D^{m} w\right\|^{2}+2 \varepsilon\left(w_{t}, w\right),
$$

By Gronwall inequality, we get

$$
y_{2}(t) \leq y_{2}(0) e^{\gamma_{2} t}=0,
$$

Thus $y_{2}(t)=0$, i.e. $u^{*}=v^{*}$, so the uniqueness is proved.

Theorem 2 According to lemma 1 and theorem 1, then the initial boundary value problem (1) - (3) has a family of global attractors

$$
A_{k}=\omega\left(B_{0 k}\right)=\underset{\tau \geq 0}{\cup_{t \geq \tau} S\left(t_{k}\right) B_{0 k}},(k=1,2,3, \cdots, 2 m),
$$

where $B_{0 k}=\left\{(u, v) \in E_{k}:\|(u, v)\|_{E_{k}}^{2}=\left\|D^{2 m+k} u\right\|^{2}+\left\|D^{k} v\right\|^{2} \leq R_{k}^{2}+R_{0}^{2}\right\}$ is a bounded absorbing set in $E_{k}$ and satisfies the following conditions:

(i) $S(t) A_{k}=A_{k}, t>0$;

(ii) $\lim _{t \rightarrow \infty} \operatorname{dist}\left(S(t) B_{k}, A_{k}\right)=0 \quad\left(\forall B_{k} \subset E_{k}\right) \quad B_{k}$ is a bounded set;

where $\operatorname{dist}\left(S(t) B_{k}, A_{k}\right)=\sup _{x \in B_{k}} \inf _{y \in A_{k}}\|S(t) x-y\|_{E_{k}}, S(t)$ is the solution semigroup generated by the initial boundary value problem (1) - (3).

Proof. It is necessary to verify the conditions (I), (II) and (III) for the existence of attractors in reference [2]. Under the condition of Theorem 1, there exists a solution semigroup $S(t): E_{k} \rightarrow E_{k}$ of the initial boundary value problem (1) - (3).

From lemma 1, we can obtain that $\forall B_{k} \subset E_{k}$ is a bounded set that includes in the ball $\left\{\|(u, v)\|_{E_{k}} \leq R_{k}\right\}$. 


$$
\left\|S(t)\left(u_{0}, v_{0}\right)\right\|_{E_{k}}^{2}=\|u\|_{H_{0}^{2 m+k}(\Omega)}^{2}+\|v\|_{H_{0}^{k}(\Omega)}^{2} \leq\left\|u_{0}\right\|_{H_{0}^{2 \mathrm{~m}+k}(\Omega)}^{2}+\left\|v_{0}\right\|_{H_{0}^{k}(\Omega)}^{2}+C \leq R_{k}^{2}+C,
$$

where $t \geq 0,\left(u_{0}, v_{0}\right) \in B_{k}$, this shows that $\{S(t)\}(t \geq 0)$ is uniformly bounded in $E_{k}$.

Furthermore, for any $\left(u_{0}, v_{0}\right) \in E_{k}$, when $t \geq \max \left\{t_{1}, t_{k}\right\}$, we have

$$
\left\|S(t)\left(u_{0}, v_{0}\right)\right\|_{E_{k}}^{2}=\|u\|_{H_{0}^{2 m+k}(\Omega)}^{2}+\|v\|_{H_{0}^{k}(\Omega)}^{2} \leq R_{k}^{2}+R_{0}^{2},
$$

Therefore,

$$
B_{0 k}=\left\{(u, v) \in E_{k}:\|(u, v)\|_{E_{k}}^{2}=\left\|D^{2 m+k} u\right\|^{2}+\left\|D^{k} v\right\|^{2} \leq R_{k}^{2}+R_{0}^{2}\right\}
$$

is a bounded absorbing set in semigroup $S(t)$.

According to the rellich kondrachov compact embedding theorem, if $E_{k}$ is compactly embedded in $E_{0}$, then the bounded set in $E_{k}$ is the compact set in $E_{0}$. Therefore, the solution semigroup $S(t)$ is a completely continuous operator, thus the global attractor family $A_{k}$ of solution semigroup $S(t)$ is obtained. Where $A_{k}=\omega\left(B_{0 k}\right)=\bigcap_{\tau \geq 0} \overline{\bigcup_{t \geq \tau} S(t) B_{0 k}}$

The prove is completed.

\section{Dimension Estimation}

In this part, we first linearize the equation into a first-order variational equation and prove that the solution semigroup $s(t)$ is Fréchet differentiable on $E_{k}$. Furthermore, we prove the decay of the volume element of the linearization problem. Finally, we estimate the upper bound of the Hausdorff dimension and fractal dimension of $A_{k}$

The initial boundary value problem (1) - (3) is linearized and rewritten into a first order variational equation

$$
\begin{gathered}
w_{t}+\varepsilon w=\theta, \\
\left.\theta_{t}-\varepsilon \theta+\varepsilon^{2} w+\Delta^{2 m} w+a(t)(-\Delta)^{m} \theta-\varepsilon a(t)(-\Delta)^{m} w+N\|\|^{m} u(t) \|_{q}^{q}\right)(-\Delta)^{m} w \\
+N^{\prime}\left(\left\|D^{m} u(t)\right\|_{q}^{q}\right)\left(\left\|D^{m} u(t)\right\|_{q}^{q}\right)^{\prime} D^{m} w(-\Delta)^{m} u=0,
\end{gathered}
$$$$
h=\left[N\left(\left\|D^{m} \bar{u}(t)\right\|_{q}^{q}\right)-N\left(\left\|D^{m} u(t)\right\|_{q}^{q}\right)\right](-\Delta)^{m} \bar{u}-N^{\prime}\left(\left\|D^{m} u(t)\right\|_{q}^{q}\right)\left(\left\|D^{m} u(t)\right\|_{q}^{q}\right)^{\prime} D^{m} w(-\Delta)^{m} u .
$$

$$
\begin{gathered}
\left.(w, \theta)\right|_{t=0}=\bar{\xi}, x \in \Omega, \\
\left.w(x, t)\right|_{\partial \Omega}=\left.(-\Delta)^{i} w(x, t)\right|_{\partial \Omega}=0,
\end{gathered}
$$

$$
\left.\theta(x, t)\right|_{\partial \Omega}=\left.(-\Delta)^{i} \theta(x, t)\right|_{\partial \Omega}=0, i=1,2, \cdots, 2 m-1, t>0 .
$$

Where $\quad \bar{\xi}=(\xi, \eta) \in E_{k} \quad, \quad(u, v)=S(t)\left(u_{0}, v_{0}\right) \quad$ is $\quad$ the solution of the initial boundary value problem (1) - (3).

Given $\left(u_{0}, v_{0}\right) \in A_{k}$, then we can get $S(t)\left(u_{0}, v_{0}\right) \in E_{k}$. It is proved that there exists a unique solution to the linearized initial boundary value problem (40) - (44) for any $(\xi, \eta) \in E_{k}$.

Lemma 2 If $S(t): E_{k} \rightarrow E_{k}$, the Frechet differential on $\eta_{0}=\left(u_{0}, v_{0}\right)$ is a linear operator $F:(\xi, \eta) \rightarrow(w, \theta)$, for any $t>0, R>0$, the mapping $S(t): E_{k} \rightarrow E_{k}$ is Fréchet differentiable on $E_{k}$, where $(w, \theta)$ is the solution of the linearized initial boundary value problem (40) - (44).

Proof. Set $\eta_{0}=\left(u_{0}, v_{0}\right) \in E_{k}, \overline{\eta_{0}}=\left(u_{0}+\xi, v_{0}+\eta\right) \in E_{k}$ and $\left\|\eta_{0}\right\|_{E_{k}} \leq R,\left\|\overline{\eta_{0}}\right\|_{E_{k}} \leq R$.

$$
\text { We define } \eta_{1}=S(t) \eta_{0}=(u, v), \overline{\eta_{1}}=S(t) \overline{\eta_{0}}=(\bar{u}, \bar{v}) \text {, }
$$
where the semigroup $S(t)$ is Lipschitz continuous on the bounded set of $E_{k}$, i.e.

$$
\left\|S(t) \eta_{0}-S(t) \overline{\eta_{0}}\right\|_{E_{k}}^{2} \leq e^{c t}\|(\xi, \eta)\|_{E_{k}}^{2}
$$

Let $(\psi, \phi)=\overline{\eta_{1}}-\eta_{1}-U=(\bar{u}-u-w, \bar{v}-v-\theta)$, then

$$
\begin{gathered}
\psi_{t}+\varepsilon \psi=\phi, \\
\phi_{t}-\varepsilon \phi+\varepsilon^{2} \psi+\Delta^{2 m} \psi+N\left(\left\|D^{m} u(t)\right\|_{q}^{q}\right)(-\Delta)^{m} \psi=-h, \\
\psi(0)=\phi(0)=0,
\end{gathered}
$$

where

By using the inner product in $E_{k}$ of Equation (47) with $\phi$, we obtain

$$
\begin{aligned}
\frac{d}{d t}\|\| D^{k} \phi\left\|^{2}+\varepsilon^{2}\right\| D^{k} \psi \|^{2} & \left.+\left\|D^{2 m+k} \psi\right\|^{2}\right)+2 \varepsilon^{3}\left\|D^{k} \psi\right\|^{2}+2 \varepsilon\left\|D^{2 m+k} \psi\right\|^{2}=2 \varepsilon\left\|D^{k} \phi\right\|^{2} \\
& +2 N\left(\left\|D^{m} u(t)\right\|_{q}^{q}\right)\left(D^{2 m+k} \psi, D^{k} \phi\right)+2\left|\left(-h,(-\Delta)^{k} \phi\right)\right|
\end{aligned}
$$

Now we deal with $h$, let $f=D^{m} u(t), \bar{f}=D^{m} \bar{u}(t)$, and deal with equation (49) with the help of differential mean value 
theorem

$$
\begin{aligned}
h=N^{\prime}\left(\left\|\varsigma_{1}\right\|_{q}^{q}\right)\left(\left\|\varsigma_{1}\right\|_{q}^{q}\right)^{\prime} D^{m}(\bar{u}-u)(-\Delta)^{m} \bar{u}-N^{\prime}\left(\|f\|_{q}^{q}\right)\left(\|f\|_{q}^{q}\right)^{\prime} D^{m}(\bar{u}-u)(-\Delta)^{m} u \\
+N^{\prime}\left(\|f\|_{q}^{q}\right)\left(\|f\|_{q}^{q}\right)^{\prime}(-\Delta)^{m} u D^{m} \psi \\
=h_{1}+h_{2}
\end{aligned}
$$

Where $\varsigma_{1}=(1-s) \bar{f}+s f, s \in(0,1), h_{1}=N^{\prime}\left(\|f\|_{q}^{q}\right)\left(\|f\|_{q}^{q}\right)^{\prime}(-\Delta)^{m} u D^{m} \psi$,

$$
h_{2}=N^{\prime}\left(\left\|\varsigma_{1}\right\|_{q}^{q}\right)\left(\left\|\varsigma_{1}\right\|_{q}^{q}\right)^{\prime} D^{m}(\bar{u}-u)(-\Delta)^{m} \bar{u}-N^{\prime}\left(\|f\|_{q}^{q}\right)\left(\|f\|_{q}^{q}\right)^{\prime} D^{m}(\bar{u}-u)(-\Delta)^{m} u .
$$

Further, let $g^{\prime}\left(\varsigma_{1}\right)=N^{\prime}\left(\left\|\varsigma_{1}\right\|_{q}^{q}\right)\left(\left\|\varsigma_{1}\right\|_{q}^{q}\right)^{\prime}$, then

$$
\begin{aligned}
h_{2} & =\left[\left(g^{\prime}\left(\varsigma_{1}\right)-g^{\prime}(f)\right)(-\Delta)^{m} \bar{u}+g^{\prime}(f)(-\Delta)^{m}(\bar{u}-u)\right] D^{m}(\bar{u}-u) \\
& =g^{\prime \prime}\left(\varsigma_{2}\right)(1-s)\left(D^{m}(\bar{u}-u)\right)^{2}(-\Delta)^{m} \bar{u}+g^{\prime}(f)(-\Delta)^{m}(\bar{u}-u) D^{m}(\bar{u}-u),
\end{aligned}
$$

where $\varsigma_{2}=\theta \varsigma_{1}+(1-\theta) f, \theta \in(0,1)$.

Therefore,

$$
\left|\left(-h,(-\Delta)^{k} \phi\right)\right|=\left|\left(-\left(h_{1}+h_{2}\right),(-\Delta)^{k} \phi\right)\right| \leq\left|\left(h_{1},(-\Delta)^{k} \phi\right)\right|+\left|\left(h_{2},(-\Delta)^{k} \phi\right)\right|,
$$

where

$$
\begin{aligned}
& \left|\left(h_{1},(-\Delta)^{k} \phi\right)\right|=\left|\left(N^{\prime}\left(\|f\|_{q}^{q}\right)\left(\|f\|_{q}^{q}\right)^{\prime}(-\Delta)^{m} u D^{m} \psi,(-\Delta)^{k} \phi\right)\right| \\
& \leq C_{5}\left\|(-\Delta)^{m} u\right\|_{\infty}\left\|D^{m+k} \psi\right\|\left\|D^{k} \phi\right\| \\
& \leq \frac{C_{6} \lambda_{1}^{m}}{2}\left\|D^{2 m+k} \psi\right\|^{2}+\frac{C_{6}}{2}\left\|D^{k} \phi\right\|^{2} \\
& \left|\left(h_{2},(-\Delta)^{k} \phi\right)\right|=\left|\left(g^{\prime \prime}\left(\varsigma_{2}\right)(1-s)\left(D^{m}(\bar{u}-u)\right)^{2}(-\Delta)^{m} \bar{u}+g^{\prime}(f)(-\Delta)^{m}(\bar{u}-u) D^{m}(\bar{u}-u),(-\Delta)^{k} \phi\right)\right| \\
& \leq\left\|g^{\prime \prime}\left(\varsigma_{2}\right)\right\|_{\infty}\left|\int_{\Omega}(-\Delta)^{m} \bar{u}\left(D^{m}(\bar{u}-u)\right)^{2}(-\Delta)^{k} \phi d x\right|+\left\|g^{\prime}(f)\right\|_{\infty}\left|\int_{\Omega}(-\Delta)^{m}(\bar{u}-u) D^{m}(\bar{u}-u)(-\Delta)^{k} \phi d x\right| \\
& \leq C_{7}\left\|D^{k} \phi\right\|\left(\int_{\Omega}\left[D^{2 m+k} \bar{u}\left(D^{m}(\bar{u}-u)\right)^{2}+D^{2 m} \bar{u} D^{k}\left(D^{m}(\bar{u}-u)\right)^{2}\right]^{2} d x\right)^{\frac{1}{2}} \\
& +C_{8}\left\|D^{k} \phi\right\|\left(\int_{\Omega}\left[D^{2 m+k}(\bar{u}-u) D^{m}(\bar{u}-u)+D^{2 m}(\bar{u}-u) D^{k}\left(D^{m}(\bar{u}-u)\right)\right]^{2} d x\right)^{\frac{1}{2}} \\
& \leq 4 C_{7}\left\|D^{k} \phi\right\|\left(\int_{\Omega}\left(D^{2 m+k} \bar{u}\right)^{2}\left(D^{m}(\bar{u}-u)\right)^{4} d x+\int_{\Omega}\left(D^{2 m} \bar{u}\right)^{2}\left(D^{m+k}(\bar{u}-u)\right)^{4} d x\right)^{\frac{1}{2}} \\
& +4 C_{8}\left\|D^{k} \phi\right\|\left(\int_{\Omega}\left(D^{2 m+k}(\bar{u}-u)\right)^{2}\left(D^{m}(\bar{u}-u)\right)^{2} d x+\int_{\Omega}\left(D^{2 m}(\bar{u}-u)\right)^{2}\left(D^{m+k}(\bar{u}-u)\right)^{2} d x\right)^{\frac{1}{2}} \\
& \leq 4 C_{7}\left\|D^{k} \phi\right\|\left(\left\|D^{m}(\bar{u}-u)\right\|_{\infty}^{2}\left\|D^{2 m+k} \bar{u}\right\|+\left\|D^{m+k}(\bar{u}-u)\right\|_{\infty}^{2}\left\|D^{2 m} \bar{u}\right\|\right) \\
& +4 C_{8}\left\|D^{k} \phi\right\|\left(\left\|D^{2 m+k}(\bar{u}-u)\right\|_{\infty}\left\|D^{m}(\bar{u}-u)\right\|+\left\|D^{2 m}(\bar{u}-u)\right\|_{\infty}\left\|D^{m+k}(\bar{u}-u)\right\|\right)
\end{aligned}
$$




$$
\begin{aligned}
& \leq 4 C_{9}\left\|D^{k} \phi\right\|\left\|D^{2 m+k}(\bar{u}-u)\right\|^{2}+4 C_{10}\left\|D^{k} \phi\right\|\left\|D^{2 m+k}(\bar{u}-u)\right\|^{2} \\
& \leq \frac{C_{9}+C_{10}}{2}\left\|D^{k} \phi\right\|^{2}+2\left(C_{11}^{2}+C_{12}^{2}\right)\left\|D^{2 m+k}(\bar{u}-u)\right\|^{4} .
\end{aligned}
$$

Combined with formula (53) - (55), it is concluded that

$$
\left|\left(-h,(-\Delta)^{k} \phi\right)\right| \leq \frac{C_{6} \lambda_{1}^{m}}{2}\left\|D^{2 m+k} \psi\right\|^{2}+\frac{C_{6}+C_{9}+C_{10}}{2}\left\|D^{k} \phi\right\|^{2}+2\left(C_{11}^{2}+C_{12}^{2}\right)\left\|D^{2 m+k}(\bar{u}-u)\right\|^{4} .
$$

Furthermore, substituting formula (56) into equation (50), the result is as follows

$$
\begin{aligned}
\frac{d}{d t}\left(\left\|D^{k} \phi\right\|^{2}\right. & \left.+\varepsilon^{2}\left\|D^{k} \psi\right\|^{2}+\left\|D^{2 m+k} \psi\right\|^{2}\right) \leq\left(\delta_{1}+C_{6}+C_{9}+C_{10}+2 \varepsilon\right)\left\|D^{k} \phi\right\|^{2}-2 \varepsilon^{3}\left\|D^{k} \psi\right\|^{2} \\
& +\left(\delta_{1}+C_{6} \lambda_{1}^{m}-2 \varepsilon\right)\left\|D^{2 m+k} \psi\right\|^{2}+4\left(C_{11}^{2}+C_{12}^{2}\right)\left\|D^{2 m+k}(\bar{u}-u)\right\|^{4} .
\end{aligned}
$$

Let $\gamma_{3}=\max \left\{\delta_{1}+C_{6}+C_{9}+C_{10}+2 \varepsilon,-2 \varepsilon, \delta_{1}+C_{6} \lambda_{1}^{m}-2 \varepsilon, 4\left(C_{11}^{2}+C_{12}^{2}\right)\right\}$, we can get

$$
\frac{d}{d t}\left(\left\|D^{k} \phi\right\|^{2}+\varepsilon^{2}\left\|D^{k} \psi\right\|^{2}+\left\|D^{2 m+k} \psi\right\|^{2}\right) \leq \gamma_{3}\left(\left\|D^{k} \phi\right\|^{2}+\varepsilon^{2}\left\|D^{k} \psi\right\|^{2}+\left\|D^{2 m+k} \psi\right\|^{2}+\left\|D^{2 m+k}(\bar{u}-u)\right\|^{4}\right) .
$$

From Gronwall inequality and Lipschitz property of (45), we can obtain

$$
\left\|D^{k} \phi\right\|^{2}+\left\|D^{2 m+k} \psi\right\|^{2} \leq e^{\left(\gamma_{3}+C_{13}\right) t}\|(\xi, \eta)\|_{E_{k}}^{4}
$$

So,

when

$$
\|(\xi, \eta)\|_{E_{k}} \rightarrow 0
$$

$\frac{\left\|S(t) \eta_{0}-S(t) \overline{\eta_{0}}-F S(t)(\xi, \eta)\right\|_{E_{k}}^{2}}{\|(\xi, \eta)\|_{E_{k}}^{2}} \leq e^{\left(\gamma_{3}+C_{13}\right) t}\|(\xi, \eta)\|_{E_{k}}^{2} \rightarrow 0$.

The prove is completed.

Theorem 3 Under the assumptions and conditions of theorem 2, then a family of global attractors $A_{k}$ of initial boundary value problem (1) - (3) has Hausdorff dimension and fractal dimension, and

$$
d_{H}\left(A_{k}\right)<\frac{1}{5} n, d_{F}\left(A_{k}\right)<\frac{6}{5} n .
$$

Proof. Let $\vartheta=R_{\varepsilon} \varphi=(u, v)^{T}, \quad \varphi=\left(u, u_{t}\right)^{T}, \quad v=u_{t}+\varepsilon u$, then $R_{\varepsilon}:\left\{u, u_{t}\right\} \rightarrow\left\{u, u_{t}+\varepsilon u\right\}$ is an isomorphic mapping. If $A_{i}(i=1,2, \cdots, 2 m)$ is the global attractor of $\{S(t)\}$, then $A_{\varepsilon i}$ is the global attractor of $\left\{S_{\varepsilon}(t)\right\}$, and they have the same dimension.

From lemma 2, we can get that $S(t): E_{k} \rightarrow E_{k}$ is
Fréchet differentiable, then the linearized first order variational equation (41) can be rewritten as

$$
\begin{aligned}
& P_{t}+\Lambda(\vartheta) P=0, \\
& P_{t}=F_{t}(\vartheta),
\end{aligned}
$$

where

$$
\begin{aligned}
& \Lambda(\vartheta)=\left(\begin{array}{cc}
\varepsilon I & -I \\
\left(N\left(\left\|A^{\frac{m}{2}} u\right\|_{q}^{q}\right)-\varepsilon a(t)\right) A^{m}+A^{2 m}+\varepsilon^{2} I+\Phi & a(t) A^{m}-\varepsilon I
\end{array}\right), \\
& I \text { is an identity operator, }-\Delta=A, \quad P=(w, \theta)^{T} \in E_{k} \text {, } \\
& \theta=w_{t}+\varepsilon w .
\end{aligned}
$$

For a fixed $\left(u_{0}, v_{0}\right) \in E_{k}$, let $\beta_{1}, \beta_{2}, \ldots, \beta_{n}$ be $n$ elements of $E_{k}$, and $w_{1}(t), w_{2}(t), \ldots, w_{n}(t)$ be $\mathrm{n}$ solutions of linear equation (59), whose initial value is $w_{1}(0)=\beta_{1}, w_{2}(0)=\beta_{2}, \ldots, w_{n}(0)=\beta_{n}$.

Therefore,

$$
\frac{d}{d t}\left\|w_{1}(t) \Lambda w_{2}(t) \Lambda \ldots \Lambda w_{n}(t)\right\|_{\Lambda E_{k}}^{2}-2 \operatorname{tr} F_{t}\left(\vartheta(\tau) \cdot Q_{n}(\tau)\left\|\beta_{1} \Lambda \beta_{2} \Lambda \ldots \Lambda \beta_{n}\right\|_{\Lambda E_{k}}=0 .\right.
$$

Furthermore, from the uniform Gronwall inequality, we can obtain

$$
\left\|w_{1}(t) \Lambda w_{2}(t) \Lambda \ldots \Lambda w_{n}(t)\right\|_{\Lambda E_{k}}^{2}=\left\|\beta_{1} \Lambda \beta_{2} \Lambda \ldots \Lambda \beta_{n}\right\|_{\Lambda E_{k}} \exp \left(\int_{0}^{t} \operatorname{tr} F_{t}(\vartheta(\tau)) \cdot Q_{n}(\tau) d \tau\right),
$$


where $\Lambda$ is the outer product, $t r$ is the trace, $Q_{n}(\tau)$ is an orthogonal projection from space $E_{k}$ to $\operatorname{span}\left\{w_{1}(t), w_{2}(t), \ldots, w_{n}(t)\right\}$.

For a given time $\tau$, let $\omega_{j}(\tau)=\left(\xi_{j}(\tau), \eta_{j}(\tau)\right)^{T}(j=1,2, \ldots n)$ be the standard orthogonal basis of spance $\operatorname{span}\left\{w_{1}(t), w_{2}(t), \ldots, w_{n}(t)\right\}$.

We define the inner product of $E_{k}$ as

$$
((\xi, \eta),(\bar{\xi}, \bar{\eta}))=\left(\left(D^{2 m+k} \xi, D^{2 m+k} \bar{\xi}\right)+\left(D^{k} \eta, D^{k} \bar{\eta}\right)\right) .
$$

To sum up, it can be concluded that

$$
\operatorname{tr}_{t}(\vartheta(\tau)) \cdot Q_{n}(\tau)=\sum_{j=1}^{N}\left(F_{t}(\vartheta(\tau)) \cdot Q_{n}(\tau) \omega_{j}(\tau), \omega_{j}(\tau)\right)_{E_{k}}=\sum_{j=1}^{N}\left(F_{t}(\vartheta(\tau)) \omega_{j}(\tau), \omega_{j}(\tau)\right)_{E_{k}},
$$

where

$$
\begin{gathered}
\left(F_{t}(\vartheta(\tau)) \omega_{j}(\tau), \omega_{j}(\tau)\right)_{E_{k}}=-\left(\Lambda(\vartheta) \omega_{j}, \omega_{j}\right) \\
\leq-\varepsilon\left\|D^{2 m+k} \xi_{j}\right\|^{2}+\frac{1}{2}\left(\delta_{1}-\varepsilon \mu_{0}\right)\left(\left\|D^{2 m+k} \xi_{j}\right\|^{2}+\left\|D^{k} \eta_{j}\right\|^{2}\right)+\frac{\varepsilon^{2} \lambda_{1}^{-2 m}}{2}\left\|D^{2 m+k} \xi_{j}\right\|^{2} \\
+\frac{\varepsilon^{2}}{2}\left\|D^{k} \eta_{j}\right\|^{2}-\lambda_{1}^{m} \mu_{0}\left\|D^{k} \eta_{j}\right\|^{2}+\varepsilon\left\|D^{k} \eta_{j}\right\|^{2}+\frac{\varepsilon}{2}\left\|D^{2 m+k} \xi_{j}\right\|^{2}+\frac{C_{14}^{2} \lambda_{1}^{-m-k}}{2 \varepsilon}\left\|D^{k} \eta_{j}\right\|^{2} \\
\leq \frac{\left(\delta_{1}-\varepsilon \mu_{0}\right)+\varepsilon^{2} \lambda_{1}^{-2 m}}{2}\left\|D^{2 m+k} \xi_{j}\right\|^{2}+\left(\frac{\varepsilon^{2}}{2}+\frac{C_{14} \lambda_{1}^{-m-k}}{2 \varepsilon}\right)\left\|D^{k} \eta_{j}\right\|^{2}+r\left\|D^{k} \eta_{j}\right\|^{2} \\
\leq-\frac{C_{15}}{2}\left(\left\|D^{2 m+k} \xi_{j}\right\|^{2}+\left\|\nabla^{k} \eta_{j}\right\|^{2}\right)+r\left\|D^{k} \eta_{j}\right\|^{2},
\end{gathered}
$$

where $\quad C_{15}=\min \left\{\left(\varepsilon \mu_{0}-\delta_{1}\right)-\varepsilon^{2} \lambda_{1}^{-2 m},-\varepsilon^{2}-\frac{C_{14} \lambda_{1}^{-m-k}}{\varepsilon}\right\}$, $r=\max \left\{2 \varepsilon, \delta_{1}\right\}$.

Owing to the $\omega_{j}(\tau)=\left(\xi_{j}(\tau), \eta_{j}(\tau)\right)^{T}, j=1,2, \ldots n$ is the standard orthonormal basis of $\operatorname{span}\left\{w_{1}(t), w_{2}(t), \ldots, w_{n}(t)\right\}$, so

$$
\begin{gathered}
\left\|D^{2 m+k} \xi_{j}\right\|^{2}+\left\|D^{k} \eta_{j}\right\|^{2}=1, \\
\sum_{j=1}^{n}\left(F_{t}(\vartheta(\tau)) \omega_{j}(\tau), \omega_{j}(\tau)\right)_{E_{k}} \leq-\frac{n C_{15}}{2}+r \sum_{j=1}^{n}\left\|\nabla^{k} \eta_{j}\right\|^{2},
\end{gathered}
$$

For almost all $t$, there are

$$
\sum_{j=1}^{n}\left\|\nabla^{k} \eta_{j}\right\|^{2} \leq \sum_{j=1}^{n} \lambda_{j}^{a-1}
$$

where $a=\frac{k}{2 m}$ and $a \in[0,1], \lambda_{j}$ is the eigenvalue of $A^{2 m}$ and $\lambda_{1}<\lambda_{2}<\cdots<\lambda_{n}$, thus

$$
\operatorname{Tr}_{t}(\vartheta(\tau)) \cdot Q_{n}(\tau) \leq-\frac{n C_{15}}{2}+r \sum_{j=1}^{n} \lambda_{j}^{a-1}
$$

Let

$$
q_{n}(t)=\sup _{\substack{\vartheta_{0} \in B_{0 k} \\ \eta_{j} \in E_{k} \\ \| D^{n} \eta_{j} \mid \leqslant 1}} \sup _{t}\left(\frac{1}{t} F_{t}\left(S(\tau) \vartheta_{0}\right) \cdot Q_{n}(\tau) d \tau\right)
$$

and

$$
q_{n}=\lim _{t \rightarrow \infty} q_{n}(t),
$$

so

$$
q_{n} \leq-\frac{n C_{15}}{2}+r \sum_{j=1}^{n} \lambda_{j}^{a-1}
$$

Therefore, the Lyapunov exponent $\kappa_{1}, \kappa_{2}, \ldots, \kappa_{n}(n>1)$ of $B_{0 k}$ is uniformly bounded, and

$$
\kappa_{1}+\kappa_{2}+\ldots+\kappa_{n} \leq-\frac{n C_{15}}{2}+r \sum_{j=1}^{n} \lambda_{j}^{a-1}
$$


such that

$$
\begin{gathered}
\left(q_{j}\right)_{+} \leq-\frac{n C_{15}}{2}+r \sum_{j=1}^{n} \lambda_{j}^{a-1} \leq r \sum_{j=1}^{n} \lambda_{j}^{a-1} \leq \frac{n C_{15}}{12}, \\
q_{n} \leq-\frac{n C_{15}}{2}\left(1-\frac{2 r}{n C_{15}} \sum_{j=1}^{n} \lambda_{j}^{a-1}\right) \leq-\frac{5}{12} n C_{15} .
\end{gathered}
$$

Further, we can get

$$
\max _{1 \leq j \leq n} \frac{\left(q_{j}\right)_{+}}{\left|q_{m}\right|} \leq \frac{1}{5}
$$

Therefore, $\quad d_{H}\left(A_{k}\right)<\frac{1}{5} n, d_{F}\left(A_{k}\right)<\frac{6}{5} n$, i.e. the Hausdorff dimension and Fractal dimension of a family of global attractors are finite.

\section{Conclusions}

On this paper, we studies the existence and uniqueness of the solution of the equation, when the order $m$ and the order $q$ of the Banach space $L^{q}(\Omega)$ meet certain conditions (Lemma 1 and Theorem 1). Then, we obtain the solution semigroup $S(t)$ generated by the equation has a family of global attractor $A_{k}$ in space $E_{k}=\left(H^{2 m+k}(\Omega) \cap H_{0}^{1}(\Omega)\right) \times H_{0}^{k}(\Omega)$ (Theorem 2). Finally, we get the Hausdorff dimension and Fractal dimension of a family of global attractors $A_{k}$ are finite (Lemma 2 and Theorem 3).

\section{References}

[1] Kirchhoff G. Vorlesungen uber mathematiche Physik [M]. stuttgart: Leipzig, B. G. Teubner, 1883.

[2] Guoguang Lin. Nonlinear evolution equation [M]. Kunming: Yunnan University Press, 2011.

[3] Xilai Dai, Guoguang Lin. Existence and uniqueness of solutions to strongly damped wave equations [J]. Journal of Yunnan University: Natural Science Edition, 2011, 33 (S2): 327-332.
[4] Narasimha K. Nonlinear vibration of an elastic string [J]. Sound Vibration, 1968, 8: 134-146.

[5] Boling Guo. Nonlinear evolution equation [M]. Shanghai: Shanghai Science and Technology Press, 1995.

[6] Zhengde Dai. Zhengde Dai Papers Collection [C]. Kunming: Yunnan University Press, 2016.

[7] Mitsuhiro N. An attractor for a nonlinear dissipative wave equation of Kirchhoff type [J]. Journal of Mathematical Analysis and Applications, 2009, 353 (2): 652-659.

[8] Zaiyun Zhang, Zhenhai Liu, Xiujin Miao. Estimate on the Dimension of Global Attractor for Nonlinear Dissipative Kirchhoff Equation [J]. Acta Applicandae Mathematicae, 2010, 110 (1): 271-282.

[9] Igor C. Longtime dynamics of Kirchhoff wave models with strong nonlinear Damping [J]. Journal of Differen- tial Equations, 2012, 252 (2): 1229-1262.

[10] Yunlong Gao, Yuting Sun, Guoguang Lin. The Global Attractors and Their Hausdorff and Fractal Dimensions Estimation for the Higer-Order Nonlinear Kirchhoff-Type Equation with Strong Linear Damping [J]. International Journal of Modern Nonlinear Theory and Application 2016, 5 (4): 185-202.

[11] Matsuyama T, Ikerata R. On the global solutions and energy decay for the wave equations of Kirchhoff-type with nonlinear damping terms [J]. Journal of Mathematical Analysis and Applications, 1996, 204 (3): 729-753.

[12] Kajitani K, Satoh A. On extension of solutions of Kirchhoff equations [J]. Journal of the Mathematical Society of Japan, 2004, 56 (2): 405-416.

[13] Lei Wan, Jinbao Dang, Guoguang Lin. Global attractor and dimension estimation of Fractional nonlinear Schrodinge equation [J]. Journal of Yunnan University: Natural Science Edition, 2010, 32 (2): 130-135.

[14] Ling Chen, Wei Wang, Guoguang Lin. The global attractors and the Hausdoff and fractal demensions estimation for the higher-order nonlinear Kirchhoff-type Equation [J]. Journal of Advances in Mathematics. 2016, 12 (09): 6608-6621.

[15] Guoguang Lin, Sanmei Yang. Hausdoff and Fractal demensions of the global attractor for the higher-order coupled Kirchhoff-type Equation [J]. Journal of Applied Mathematics and Physics. 2017, 5: 2411-2424. 